\title{
DIEGO DE LOS SANTOS Y ÁVILA: UN NOMBRE PARA DOS ARQUTTECTOS
}

\section{Rogelio RuIz GOMAR}

\section{A Martha Fernández}

Diego de los Santos y Ávila es el nombre de uno de los arquitectos que estuvieron activos en la Nueva España. No fue ciertamente uno de los más geniales ni destacados, ${ }^{1}$ pero faltaríamos a la verdadd si dijéramos que se trata de un artífice totalmente desconocido o mediocre. Al parecer, fue hasta con los estudios que dedicara don Francisco de la Maza al tribunal de la Inquisición que su nombre salió a la luz, pero lo hizo ni más ni menos que como maestro mayor de las obras de la temida institución. ${ }^{2}$ Tiempo después, gracias a un artículo de Eduardo Báez sobre las obras en la iglesia de San Francisco, nos enteramos de que a principios del siglo XvIII intervino al lado de Feliciano Cabello en la reedificación de la misma, hasta el año de 1712 en que murió ${ }^{3}$ Y merced al trabajo de Efraín Castro sobre el desarrollo arquitectónico del edificio de Palacio Nacional, sabemos que, en su calidad de "veedor del gremio" de arquitectos, se tomó su parecer sobre el estado que guardaba la parte vieja del Palacio de los Virreyes a principios de esa misma centuria decimoctava, y que participó en la elaboración de la nueva planta para la reconstrucción del mismo. ${ }^{4}$ Finalmente, con las anteriores noticias y otras que sobre él localizó en el Archivo de Indias, de la ciudad de Sevilla, la maestra Martha Fernández armó la mejor revisión

\footnotetext{
${ }^{1}$ Como se desprende del hecho de que ni siquiera lo menciona don Manuel Toussaint, piedra angular de los estudios del arte de la Nueva España, Arte colonial en México (la. ed,, 1948). México, Universidad Nacional Autónoma de México, Instituto de Investigaciones Estéticas, y de que no figura aún en la última edición "corregida y aumentada" del Diccionario Porría de historia, biografía y geografía de México, 5a ed., 3 vols., México, Editorial Porrúa, 1986, 3 vols.

${ }^{2}$ Francisco de la Maza, "El proyecto para la capilla de la Inquisición", Anales del Instituto de Investigaciones Estéticas, año III, núm. 12 (México, 1945), pp. 19-26, y El Palacio de la Inquisición, México, Universidad Nacional Autónoma de México, 1951.

${ }^{3}$ Eduardo Báez Macías, "Noticias sobre la construcción de San Francisco de México (1710-1716)", Anales del Instituto de Investigaciones Estéticas, año XII, núm 44 (México, 1975), pp. $31-42$.

${ }^{4}$ Efraín Castro Morales, "Evolución arquitectónica", en Palacio Nacional, México, Secretaría de Obras Públicas, 1976, pp. 90-96 y 301.
} 
con que contamos sobre la vida y obra de este arquitecto, al consagrarle un capítulo en su muy completo estudio sobre los "maestros mayores" de la ciudad de México, estudio del que me he servido ampliamente para elaborar esta nota ${ }^{5}$ Por todo ello, ahora sabemos que la actividad profesional de Diego de los Santos y Ávila, al igual que la de la mayoría de los arquitectos de la época virreinal, fue variada: lo mismo le encontramos interviniendo en obras o proyectos importantes, que en trabajos francamente irrelevantes; tanto haciendo reparos que participando en reconocimientos, elaborando dictámenes que haciendo avalúos.

Convendría recordar, tal y como lo ha demostrado la maestra Fernández, que el nombramiento de "maestro mayor" adquirió más consideración e importancia a partir del siglo XVII en el México virreinal, y que si, por un lado, el cargo implicaba enorme responsabilidad, por el otro confería no poco prestigio al artífice que lo detentaba, toda vez que de alguna manera venía a ser el reconocimiento público a su capacidad. $Y$, evidentemente, mientras más importante era la obra -pongamos por caso la Catedral o el Palacio de los Virreyes-, más lustre recibía el responsable de la misma.

Así las cosas, parece claro que el nombramiento de "maestro mayor de las obras del Santo Oficio" no podía recaer en cualquier alarife, ni el designado pasar completamente inadvertido. Pues bien, tal fue el nombramiento que ostentó Diego de los Santos y Ávila entre los años de 1657 y 1663, y que, a su tiempo, obtendrían maestros como Juan Montero y Pedro de Arrieta. Y por más de que no se llegaron a realizar los proyectos que Diego de los Santos preparó para dicha institución -la reedificación de las cárceles y la erección de una capilla-, es indudable que el solo hecho de haber sido elegido para intervenir en ellos mucho ha contribuido para que su nombre se considerara digno de ser rescatado del olvido y adquirir un resplandor adicional.

Sin embargo, hasta ahora todos hemos venido creyendo que se trataba de un solo arquitecto. El doctor Francisco de la Maza, atendiendo al hecho de que su actividad se extendía desde 1657 , "como primera fecha conocida" -en realidad desde 1652-, hasta 1712, sólo acertó a expresar que "su vida fue, pues muy larga". ${ }^{6}$ La maestra Fernández, por su parte, encontró sospechosa tan espaciada actividad, pero sin ninguna base para proponer nada en concreto, sólo apuntó: "Su longevidad es sorprendente."

\footnotetext{
${ }^{5}$ Martha Fernández, Arquitectura y gobiermo virreinal. Los maestros mayores de la ciudad de México. Siglo XVII, México, Universidad Nacional Autónoma de México, Instituto de Investigaciones Estéticas, 1985 (Estudios y Fuentes del Arte en México, XLV).

${ }^{6}$ Francisco de la Maza, "El proyecto para la capilla...", p. 26.

${ }^{7}$ Martha Fernández, op. cit., p. 195, nota 3.
} 
En tal estado de cosas, no quedaba sino intentar avanzar en la dirección trazada por el doctor de la Maza: "Muchas obras deben haberse ejecutado por sus manos en cincuenta y cinco años de trabajo -60 en realidad-, que debemos exhumar de los archivos..."

Pues bien, es de un archivo, concretamente del muy rico repositorio que conforma el actual Archivo del Sagrario Metropolitano, de donde he exhumado curiosas e inesperadas noticias que han venido a abrir nuevas perspectivas para el estudio de este arquitecto. Me apresuro a advertir que dichas noticias, más que venir a enriquecer el conocimiento de su actividad profesional, mismo que lateralmente sí se ve afectado, han venido a esclarecer un punto que no estaba siquiera en discusión, y que resulta de enorme trascendencia: bajo el nombre de Diego de los Santos y Ávila se ha venido englobando, en realidad, la actividad de dos arquitectos distintos, aunque intimamente relacionados entre sí por lazos familiares: Diego de los Santos, padre, y Diego de los Santos, hijo, a quienes, para una más cabal identificación, bien pudiéramos empezar a designar, de acuerdo con la costumbre, como Diego de los Santos y Ávila, el viejo, y Diego de los Santos y Ávila, el mozo.

Para llegar a esta conclusión me baso en un documento que considero definitivo: una partida de amonestación datada en marzo de 1686, que nos deja saber del matrimonio que pretendía contraer

Diego de los Sanctos y Avila, natural y vecino de esta ciudad, hijo legítimo de Diego de los Sanctos y Avila y de doña Josepha Ortis y Villegas con Sebastiana María de Chabera, natural y vecina de esta dicha ciudad, huérfana. ${ }^{9}$

Está claro, pues, que se trata de padre e hijo con un mismo nombre. Pero la confusión crece cuando caemos en la cuenta de que, además de compartir el nombre, ambos desarrollaron la misma profesión. Así, Diego de los Santos y Ávila, el viejo, fue el "maestro mayor" del Santo Oficio a mediados del xviı, y Diego de los Santos y Ávila, el mozo, fue quien intervino en el reconocimiento de las obras de Palacio, el que participó en el arranque de las obras de reedificación del templo de San Francisco y, evidentemente, quien murió en 1712 .

Las siguientes noticias extraídas del mismo archivo, aunque igualmente de carácter extra-artístico, vienen a reforzar lo dicho.

${ }^{8}$ Francisco de la Maza, "El proyecto...", p. 26

${ }^{9}$ Archivo del Sagrario Metropolitano. Libro 12 de amonestaciones de españoles, 1680-1687, f. 131 v. El sacerdote que los casó, como consta en una nota marginal y en la correspondiente partida matrimonial (vid infra, nota 19) fue el licenciado don Gregorio de Iseta. 
Diego de los Santos y Ávila, el viejo. contrajo matrimonio con Josefa Ortiz de Villegas en noviembre de $164 i$. Pero conviene advertir que con base en la pasmosa flexibilidad para enu. iciar los nombres en la época, a nuestro arquitecto -a quien en otras ocasiones se le habría de consignar simplemente como "Diego de Ávila" o "Diego de los Santos", hecho que me crearía serias confusiones con otros varios homónimos- se le registró en la partida matrimonial correspondiente como "Diego de Montealegre y Abila" y a ella tan solo como "Josepha de Villegas". ${ }^{10}$ De este enlace habrían de nacer cuando menos cuatro hijos. El 19 de diciembre de 1649 fue llevada a bautizar la primera hija, Andrea: ${ }^{11}$ dos años más tarde, el 12 de marzo de 1651 , era bautizada María; ${ }^{12}$ un año y meses después, el 10 de agosto de 1652, Josefa; ${ }^{13}$ y finalmente, después de tres hijas, un varón al que, en octubre de 1653, impusieron el mismo nombre del padre, hecho normal pero que mucho contribuiría a la futura confusión; y es que, si bien no hay forma de comprobarlo, creo que este niño no es otro que el futuro Diego de los Santos y Ávila, el mozo. La partida correspondiente de bautizo reza así:

\section{Diego}

En primero de octubre de 1653 años, con licencia del cura semanero bauticé a Diego, hijo de Diego de los Sanctos y de Josepha de Villegas; fueron sus padrinos Antonio Gutiérrez de Coca y doña Isabel Muñoz.

El licenciado Pedro
Mexia

\author{
Bachiller Nicolás de \\ Villalobos (?) ${ }^{14}$
}

Desafortunadamente seguimos ignorando del primer. Diego de los Santos muchas cosas. De acuerdo con lo asentado en su partida matrimonial, era natural de la ciudad de México, pero nos sería muy útil, además de poder precisar otras cosas, saber cuándo nació o quiénes fueron sus padres. Este

${ }^{10}$ ASM. Libro 7 de matrimonios de españoles, 1646-1657, f. 53 v. Una nota marginal nos informa que la ceremonia de la velación se llevó a cabo en la iglesia de la Santísima Trinidad un año después, el 17 de noviembre de 1659, poco antes de que naciera la primera hija.

${ }^{11}$ El que ofició fue el maestro fray Juan de Ayrolo "comendador de la orden de Nuestra Señora de la Merced", y fue su padrino el capitán Gabriel López Páramo. ASM. Libro 16 de bautismos de españoles, 1647.1652, f. 165.

${ }^{12}$ El celebrante fue el mismo fray Juan de Ayrolo, pero ahora el padrino fue Simón de Haro ASM Ibidem, f. 262 v.

${ }^{13}$ Ofició fray Andrés de Jér ez y el padrino fue "el reverendo padre maestro fray Gerónimo de Andrade, Provincial del Orden de Nuestra Señora de la Merced". ASM Libro 17 de bautismos de españoles, $1652-1655, \mathrm{f} .30 \mathrm{~V}$

${ }^{14}$ ASM Ibidem, f 108. 
último punto esperaba esclarecerlo localizando la correspondiente partida de amonestación, pero mis pesquisas hasta ahora han sido infructuosas. Con todo, acaso haya sido hijo del matrimonio de Diego de los Santos y Josefa Rodríguez que en 1639 llevó a bautizar una hija, cuyo padrino fue el arquitecto Melchor Pérez de Soto; o, más probablemente aún, hijo de aquel otro formado por Diego de los Santos y doña Ana Ramos de Montealegre, cuya hija, Antonia de los Santos - natural de la ciudad de Puebla, y vecina de esta de México más de 10 años-, pretendía casarse, en julio de 1652, con Nicolás Carrillo. ${ }^{15}$ Es muy probable que la conformidad en el nombre de los padres no pase de ser una mera coincidencia, al igual que la circunstancia de que el padrino en el primer caso también hubiese sido un arquitecto; pero creo que, por ahora, conviene retener ambas posibilidades.

Por otro lado, si consideramos que fue a finales de 1648 que se verificaba su matrimonio, y que para entonces contaba con alrededor de unos 20 años, no creo estar muy lejos de la verdad si propongo que debió nacer hacia el año de 1625. Sea como fuere, el primer dato cierto que tenemos de él es el de que en febrero de 1646 fungía como padrino de bautizo de Inés, una hija del matrimonio de Luis Infante de Rioja y María de la Candelaria. ${ }^{16}$

A partir de su boda con Josefa de Villegas, ésta empieza a figurar con más frecuencia. Así, ambos aparecen como padrinos de bautizo de varios niños: ${ }^{17}$ ahora bien, el que algunos de ellos resulten "hijos de la Iglesia", esto es, hijos naturales, tenidos fuera de matrimonio o simplemente abandonados, parece indicar, aunque sea de manera indirecta, que era el suyo un matrimonio bien avenido, con sólidos principios morales, y dispuesto a ayudar al prójimo poniendo en práctica profundos principios de caridad cristiana, toda vez que el vínculo o parentesco espiritual inherente a la aceptación de ser padrino de bautizo implicaba convertirse en los "padres espirituales" de dichas criaturas, lo cual, para la época colonial significaba, definitivamente, una responsabilidad mucho más seria y fuerte que la que se reconoce hoy en día.

${ }^{15}$ ASM. Libro 13 de bautismos de españoles, 1634-1639, f 407, y Libro 6 de amonestaciones de españoles, 1646-1652, f: 67, respectivamente.

${ }_{17}^{16}$ ASM. Libro 15 de bautismos de españoles, 1644-1647, $\mathrm{f} 165$.

${ }^{17}$ De Josefa, hija de Juan de la Torre y de doña Ana López Hidalgo, el 31 de julio de 1650 ASM. Libro 16 de bautizos..., f. 217; de Constanza, hija de la Iglesia, el 13 de noviembre de 1661, ASM Libro 20 de bautismos de españoles, 1660-1663, f. 111v; de Lucía, hija de la Iglesia, el 2 de enero de 1662, Ibidem, f. 122; de María, hija de Andrés de Castro y Antonia Ortiz, Ibidem, f. $151 v$ (acaso la madre de la niña haya sido cuñada de nuestro arquitecto, pues tiene el mismo apellido que su esposa); de Joseph, hijo de Juan de Velarde y doña María de Peralta, ASM. Libro 24 de bautismos de españoles, 1672-1674, f. 30v; y de Bernardo de San Dimas, hijo de la Iglesia, en junio de 1673, Ibidem, f. 76. 
De Diego de los Santos y Ávila, el mozo, tengo las siguientes noticias. Nació en la ciudad de México hacia los últimos dias del mes de septiembre de 1653, toda vez que, como quedó asentado líneas atrás, fue llevado a bautizar en la parroquia catedralicia el primero de octubre de ese año. Ignoramos cómo y dónde transcurrió su infancia y adolescencia, pues no volvemos a saber nada sobre él hasta marzo de 1686 en que, cumplidos los 32 años y cinco meses de edad, le encontramos pretendiendo contraer el primero de sus tres matrimonios. ${ }^{18} \mathrm{La}$ ceremonia se verificó dos semanas después. ${ }^{19}$ Como consta en la mencionada partida de amonestación, su primera esposa, Sebastiana María de Chavira, era natural de la ciudad de México y aunque es registrada como huérfana, no sería nada extraño que hubiera sido descendiente de alguno de los arquitectos de este apellido, Francisco o Andrés de Chavira, activos en esa centuria decimoséptima en la ciudad de Querétaro ${ }^{20}$ Con ella habría de procrear dos hijos: José Pablo, nacido en los primeros días de 1687,,$^{21}$ y Francisco Pantaleón, nacido en julio de $1689 .{ }^{22}$ A la muerte de la esposa pasó a segundas nupcias con Melchora de Espíndola. ${ }^{23}$ Ello ocurrió en mayo de 1693, pero para febrero del siguiente año, la misma fallecía, seguramente al tratar de darle un hijo. Nuestro arquitecto quedaba viudo otra vez; empero, no habría de estarlo por mucho tiempo, pues a escasos tres meses, esto es para finales del mes de mayo de ese mismo año de 1694, se corrían las amonestaciones para el que sería su tercer y último matrimonio. El nombre de su tercera esposa fue Matiana de Góngora y Pastrana -en otros documentos se le menciona

\footnotetext{
${ }^{18}$ La última de las tres amonestaciones que disponía el Concilio de Trento está datada el 3 de ese mes de marzo, vid supra nota 9 .

${ }^{19}$ En la nota marginal a la partida de amonestación se da la fecha del 25 de marzo, pero la verdad es que el enlace se llevó a cabo el 19 de ese mismo mes, según consta en la partida matrimonial: "En 19 de marzo de 1686 años, con licencia del bachiller don Juan de Padilla, cura teniente de esta santa iglesia, yo, el bachiller don Gregorio de Iseta, desposé a Diego de los Santos y Ávila con María de Chabela, siendo testigos don Agustín de Serbantes y don Joseph Abiles, presbítero, presentes. Bachiller don Juan de Padilla. Bachiller Gregorio de Ysetta (rúbricas). ASM Libro 11 de matrimonios de españotes, 1680-1688, f. 275v.

${ }^{20}$ Mina Ramírez Montes, "Arquitectura civil", en Querétaro. Ciudad barroca, México, 1988, p. 62

${ }^{21}$ Fue bautizado el 30 de ese mes y año. La María de los Santos y Ávila que aparece como madrina, era seguramente hermana del arquitecto ASM Libro 28 de bautismos de españoles, $1685-1688$, f $126 \mathrm{v}$

${ }_{22}$ Fue su padrino don Pablo de Vargas Valadés. ASM Libro 29 de bautismos de españoles, $1688-1691$, f $117 \mathrm{v}$

${ }^{23}$ Ella era "natural de Calimaya", vecina de la ciudad de México "de ocho años a esta parte" y era hija de Tomás de la Cruz y Juana de Espíndola Quizá pertenecía a la feligresía de la parroquia de la Santa Veracruz, pues en una anotación marginal se alude a que se "Ilevó certificación a los curas de la Veracruz". ASM Libro 13 de amonestaciones de españoles, $1688-1694$, f. $113 \mathrm{v}$
} 
con el apellido de Valderrama-;24 el matrimonio se verificó el 6 de junio, y cabe destacar que en dicha ceremonia fungió como testigo Pedro de Arrieta. ${ }^{25}$

De este enlace habrían de nacer cuando menos otros cuatro hijos: María de los Dolores, que fue llevada a bautizar el 25 de abril de 1700 y fueron sus padrinos Felipe de Roa y María Teresa de Roa; ${ }^{26}$ Francisco Hipólito Casiano, que nació en agosto de $1701 ;{ }^{27}$ Diego Manuel, en marzo de $1704,{ }^{28}$ y Ana Lorenza Bernarda, hasta agosto de $1710 .^{29}$

Así las cosas, no queda la menor duda de que a él corresponde la partida de defunción datada el 5 de noviembre de 1712; él era quien con su muerte dejaba viuda a doña Matiana de Góngora, el que vivía en la Santísima Trinidad y el que fue enterrado en San Francisco. ${ }^{30}$

Noticias de su familia sólo tengo las de que su hija María pretendía casarse con Bernardo González de Bustamante, en junio de 1722, ${ }^{31}$ y de que su viuda, Matiana de Góngora, habría de sobrevivirle más de 20 años, pues murió hasta noviembre de $1735 .^{32}$

La pregunta que ahora urge responder es la de cuándo murió Diego de los Santos el viejo y no tanto por mero prurito de exactitud, sino porque de este dato depende el poder deslindar, dentro de la actuación profesional que se conoce, qué obras corresponden al viejo y cuáles al mozo. Desafortunadamente no he encontrado en el archivo de la parroquia catedralicia la noticia de su deceso; chabrá fallecido fuera de la ciudad de México? Difícil saberlo. Pero a falta del dato exacto, resulta de gran ayuda el hecho de que en la partida de defunción de su esposa, Josefa Ortiz de Villegas, quien murió el 14 de marzo de 1679 , se le registre ya como "viuda

\footnotetext{
${ }^{24}$ Ella era natural y vecina de la ciudad de México e hija de Juan de Góngora y de Antonia de Cárdenas. ASM Libro 14 de amonestaciones de españoles, 1694-1701, f. $2 \mathrm{~V}$.

${ }^{25}$ ASM. Libro 12 de matrimonios de españoles, 1688-1694, f. 287v. La ceremonia de velación fue ese mismo día en el Hospital de San Lázaro.

${ }^{26}$ ASM Libro 32 de bautismos de españoles, 1698-1701, f. 161

${ }^{27}$ Fue madrina María de Góngora de Valderrama, su abuela o tía. ASM. Libro 33 de bautisnos de españoles, $1701-1705$, f $30 \mathrm{v}$.

${ }^{28}$ Ibidem., f $299 \mathrm{v}$. Fueron sus padrinos el licenciado don Joseph Nolasco de Herrera, abogado de la Real Audiencia, y doña María Francisca de Esquivel.

${ }_{29}$ Fue su padrino "el bachiller don Pedro de Castrillo, presbítero" y ofició el mercedario fray Pedro de Vega; ASM. Libro 35 de bautismos de españoles, $1707-1712$, f. $177 \mathrm{v}$

${ }^{30}$ ASM. Libro 6 de defunciones de españoles, 1707.1714, f. 321v. La fecha de su muerte ya la habian registrado Eduardo Báez, op cit., p. 34 y la maestra Martha Fernández, op. cit., p. 195; a esta última debemos, además, la transcripción de la dicha partida de defunción: Documento $\mathrm{XI}$, p. 315

${ }^{31}$ ASM Libro 18 de amonestaciones de españoles, 1719-1727, f. 68.

32 ASM Libro 11 de defunciones de españoles, 1734-1737, f. 96v. Vivía en "la Pila del barrio de la Santísima Trinidad".
} 
de Diego de los Santos". ${ }^{33}$ Luego, si el padre murió antes de marzo de 1679, el "Diego de los Santos y Ávila" que aparece con Andrea de los Santos en agosto de ese mismo año como padrino de bautizo de un niño ${ }^{34}$, es necesariamente el hijo. Pero ¿es el padre o el hijo el que, tres años atrás, en febrero de 1676, había fungido como padrino de otro niño? ${ }^{35}$

Una vez establecida, aunque sea en forma vaga, la fecha de muerte del padre, podemos pasar a intentar determinar cuáles de las actividades profesionales que hasta ahora se entendían como de uno solo, corresponden al padre y cuáles al hijo. Para ello nos vamos a servir de la completa relación que ofrece la maestra Martha Fernández, a cuyo estudio remitimos al interesado para mayor y más detallada información. ${ }^{36}$

Fue Diego de los Santos y Ávila, el viejo, quien en 1652 figura como obrero en la edificación del convento de la Concepción y quien, junto con Nicolás de la Piedra, emitió opinión favorable con relación a la obra que el maestro Juan Serrano había ejecutado en la catedral de México; ${ }^{37}$ pero fue Diego de los Santos, el mozo, quien, en 1689, fue convocado junto con otros catorce maestros para dictaminar sobre lo que habría de realizarse en el mismo edificio catedralicio, una vez que se terminara la portada lateral. $^{38}$

Pero prosigamos con orden. Fue el padre quien en 1658 reconoció, junto con Luis Gómez de Trasmonte y Rodrigo Díaz de Aguilera, los reparos que eran necesarios en el edificio de Palacio para corroborar la memoria que había elaborado el primero ${ }^{39} \mathrm{Al}$ lado de los anteriores y de Pedro Ramírez dio su parecer, en febrero de 1660, acerca de la planta elaborada por Vicente Barroso de Escayola para la catedral de Valladolid, hoy Morelia. ${ }^{40}$

Fue también el padre quien, como "maestro mayor" del Santo Oficio, desarrolló entre 1657 y 1663 una intensa labor para dicha institución. Empezó reconociendo el estado que guardaban las cárceles secretas, reco-

\footnotetext{
${ }^{33}$ ASM Libro 1 de defunciones de españoles, 1671-1680, f. 290v Vivía en "la Puente de la Leña" y se enterró en la iglesia de la Merced.

${ }^{34}$ De Antonio Eusebio, hijo de Agustín Dorantes y María Balli. ASM. Libro 26 de bautismos de españoles, 1679-1682, f 41

${ }^{35}$ De José Nolasco, "hijo de la Iglesia", en febrero de 1676; ASM. Libro 25 de bautismos de españoles, 1674-1679, f 113.

${ }^{36}$ Martha Fernández, op cit., pp. 195-208; pero mejor aún la "relación cronológica", pp. 276-278.

37 Ibidem, pp. 198, 196-197 y 276

${ }^{38}$ Ibidem, pp 197 y 277.

${ }^{39}$ Efraín Castro Morales, op cit., pp. 62 y 301.

${ }^{40}$ Martha Fernández, op. cit., p. 197
} 
mendando apuntalar zonas en tanto se sustituían techos. En vista de la gravedad que guardaban y en respuesta a la petición de los inquisidores, en octubre de 1657 elaboró la planta y presentó presupuesto para las obras de las cárceles nuevas; proyecto en el que, como apunta la maestra Fernández, se encuentra valiosa información técnica de los procedimientos constructivos ${ }^{41}$ Aunque el doctor de la Maza da a entender que la obra -rematada en Pedro Durán- se llevó a cabo, ${ }^{42}$ la verdad es que no fue así, como se desprende del hecho de que todavía en junio de 1663 el propio arquitecto entregaba unas explicaciones sobre su propuesta anterior, y el que, un mes más tarde, Rodrigo Díaz de Aguilera y Rodrigo Bernal de Ortega emitieran serias críticas a dicho proyecto. ${ }^{43}$ Gracias a la documentación manejada por la maestra Fernández podemos ver que en ese lapso Diego de los Santos se encargó de una serie de reparos, pero también que se vio envuelto en varios problemas. Al exigirle la institución que rindiera cuentas, así de lo realizado como del material empleado, y ser detectadas supuestas anomalías, el arquitecto fue condenado a pagar el daño que las instalaciones habían sufrido por no haber hecho oportunamente las reparaciones pertinentes, se le dio un corto plazo para hacer dichas reparaciones y se le impuso una multa de 50 pesos. Al parecer todo se aclaró, pero hasta 1661: la obra realizada equivalía a lo que había recibido, y una de las partidas que se decía le correspondía, en realidad se había librado a Barroso y Escayola. ${ }^{44}$ En el ínterin, Diego de los Santos fue requerido para elaborar los planos de la nueva capilla que pretendía construir el Santo Tribunal. Nuestro arquitecto, como sabemos gracias al trabajo de don Francisco de la Maza, entregó dichos planos el 14 de febrero de $1659 .{ }^{45}$ En lo que se equivocó don Francisco es en lo de que la nueva capilla "estaría dedicada al mártir del Santo Oficio, San Pedro Arbués, primer inquisidor de Cataluña", cuya beatificación se verificaría cinco años después, en 1664, y no sería canonizado sino hasta 1869 . En realidad dicha capilla iba a estar consagrada a San Pedro Mártir, santo dominico italiano, también conocido como San Pedro de Verona, quien fuera nombrado Inquisidor General por Gregorio IX y fuera martirizado en 1252.

De acuerdo con el proyecto presentado, la capilla iba a estar ubicada en la esquina del edificio "donde antes era el tribunal viejo", ubicada de oriente a poniente, sobre la calle de la Perpetua; iba a estar constituida por

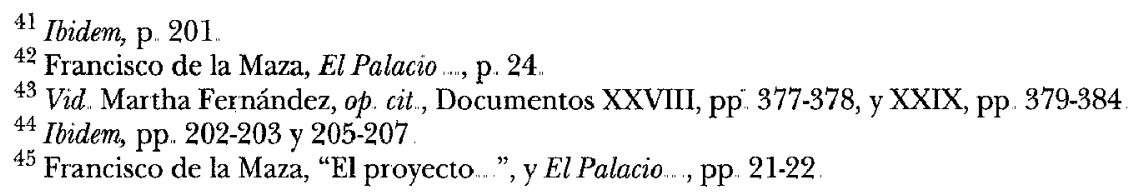


cinco bóvedas de lunetos "por ser las más hermosas y fuertes", cúpula, dos torrecillas, un amplio coro con dos tribunas laterales con sus respectivas celosías, y bajo la nave una amplia cripta para que sirviera de "entierro de la cofradía" del mencionado San Pedro Mártir. Finalmente, iba a lucir una portada "muy suntuosa de cantería" que se "ha de poner donde hoy está la entrada del Santo Oficio y la que hoy está se ha de pasar más adelante", esto es, sobre la calle de Santo Domingo; portada que conocemos gracias al dibujo que del propio autor se conserva, y sobre la que han emitido acertados juicios el propio doctor de la Maza y don Diego Angulo, mismos que oportunamente transcribió la maestra Fernández, por lo que considero innecesario volverlos a recordar ${ }^{46}$

Lamentablemente este proyecto tampoco se llevó a cabo. Como hemos apuntado, en junio de 1663 entregaba unas aclaraciones al proyecto anterior de las cárceles, mismo que fue severamente criticado por Díaz de Aguilera y Rodrigo Bernal. Quizá esto fue lo que provocó el rompimiento entre Diego de los Santos y el Tribunal de la Inquisición; lo cierto es que justo después de este incidente se menciona a Rodrigo Bernal de Ortega como "maestro de las obras de este Santo Oficio", ${ }^{47}$

En 1667 realizó varios reconocimientos a las obras del desagüe,,$^{48}$ y en 1668 se ausentó de la ciudad de México para ver las obras que se realizaban en la catedral de Oaxaca. ${ }^{49}$

Acaso su última intervención sea el compromiso que aceptó para edificar una casa particular en octubre de 1673. Como señala la maestra Fernández, "El arquitecto realizó la planta y comenzó la obra, pero la dejó inconclusa". ${ }^{0}$ ¿Detrás de esto no estará la incapacidad física o la muerte del maestro?

En relación con el segundo Diego de los Santos y Ávila, la primera actividad profesional conocida parece ser su participación, en mayo de 1688, al lado de los maestros Diego Martín de Herrera y José de los Santos -¿pariente suyo?-, en una reunión con los religiosos del convento de la Merced; reunión en la que se acordó labrar unas casas para arrendar, frente a la puerta de campo, edificar sobre ellas el oratorio del noviciado nuevo, y guanecer la cerca en que habría de quedar la puerta reglar de dicho convento. ${ }^{51}$

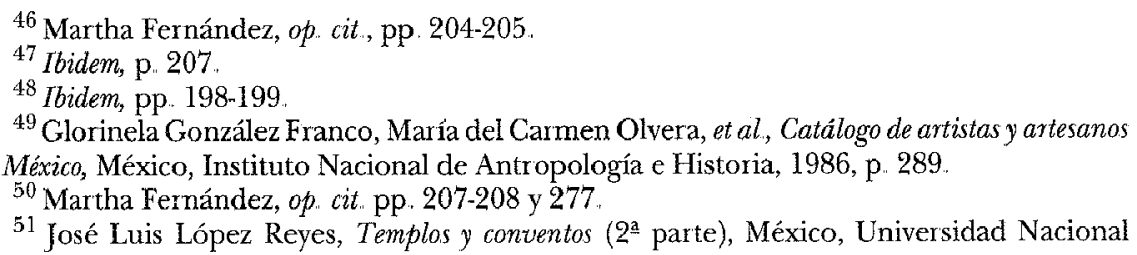


A continuación vendría su participación, en 1689, al lado de catorce consagrados maestros, respecto a decidir qué hacer en la catedral metropolitana después de que se concluyera la portada del crucero del lado izquierdo. ${ }^{32}$

Tiempo después suscribió, junto con Felipe de Roa, Antonio Jirón y Pedro de Arrieta, una declaración tocante a la construcción del cimborrio de la catedral de Valladolid, Morelia, obra que estaba a cargo del maestro Juan de Silva Carrión; dicha declaración se insertó en el mandamiento del virrey al cabildo de aquella catedral para que se prosiguiera la obra. ${ }^{53} \mathrm{En}$ 1702 reconoció y declaró sobre la limpieza de la acequia de la ciudad de México "que llaman de la Merced" ${ }^{54}$ A principios de ese mismo año, el arquitecto Felipe de Roa, a la sazón maestro mayor de las obras de Palacio y compadre suyo solicitó que se hiciese un reconocimiento del estado que guardaba la parte vieja del mismo. Para acompañar a Roa fueron convocados nuestro arquitecto y Pedro de Arrieta, en su calidad de veedores del gremio, y el maestro Marcos Antonio Sobrarías. El resultado fue el detallado informe entregado el 16 de febrero en el que advertían el general mal estado del edificio; curiosamente, nuestro artífice participó en su elaboración pero parece que no lo suscribió. ${ }^{55}$

Aunque ciertas partes se iban apuntalando, el riesgo de ruina continuaba; por ello seis años más tarde, en agosto de 1708, fueron designados Diego de los Santos, Antonio Mejía y Marcos Antonio Sobrarías para que, junto con Felipe de Roa, quien seguía dirigiendo las obras en Palacio, hiciesen nuevo reconocimiento a la Sala de la Real Audiencia, los Tribunales de Cuentas, la Sala del Crimen, Cárcel de Corte, Juzgado de Bienes de Difuntos, Cancillería, etc. Como su conclusión fue la de que no valía la pena continuar haciendo reparaciones al inmueble, se determinó pedirles a los mismos maestros que hicieran una nueva planta, la cual, pese a la muerte del maestro mayor, Felipe de Roa, entregaron el 20 de febrero de 1709 junto con el costo estimado que significarían las obras. ${ }^{56}$ A solicitud del

\footnotetext{
Autónoma de México, Instituto de Investigaciones Estéticas, 1985 (Catálogo de Documentos de Arte en el Archivo General de la Nación, México, 9), p.8: 048

${ }_{52}$ Martha Fernández, op. cit., pp. 197 y 277

${ }^{53}$ El mandamiento del virrey está fechado el 30 de julio de 1703. Vid. Mina Ramírez Montes, Reales Cédulas Duplicadas, México, Universidad Nacional Autónoma de México, Instituto de Investigaciones Estéticas, 1986 (Catálogo de Documentos de Arte en el Axchivo General de la Nación, México. 10), p. 37: 249.

${ }_{55}^{54}$ Martha Fernández, op. cit., pp. 199 y 277.

${ }^{55}$ Efraín Castro Morales, op cit.., p. 90, y Documento 36, pp . 229-230, y Martha Fernández, op. cit., p. 199-200 y 277 .

${ }_{56}$ Efraín Castro Morales, op cit, pp. 92-96, y Documento 39, pp. 231-232.
} 
decano de los oidores, Diego de los Santos y el nuevo maestro mayor de las obras de Palacio, Francisco Antonio de Roa, pasaron en abril de ese mismo año a reconocer las casas principales del Marqués del Valle, para ver si provisionalmente se podían instalar en ellas los Tribunales de la Audiencia; en abril de ese año entregaron su informe en sentido negativo. ${ }^{57}$

Sólo resta recordar la participación de Diego de los Santos, el mozo, en las obras para la reedificación del templo del convento de San Francisco de la ciudad de México. Como la iglesia se encontraba para 1710 en mal estado, los religiosos decidieron consultar con los maestros Feliciano Cabello, Manuel Martínez de Herrera y Diego de los Santos lo que se podía hacer. Su dictamen fue que era inútil pretender hacer reparos y que sólo cabía hacerla de nuevo. ${ }^{58}$ Nuestro arquitecto y Feliciano Cabello fueron electos responsables para "maestrear" la obra con sueldo de un peso diario cada uno, ${ }^{59}$ la intervención de Diego de los Santos abarcó del 4 de noviembre de ese año de 1710 , en que se iniciaron los trabajos, hasta su muerte acaecida el 5 de noviembre de 1712 , y en que continuó solo Feliciano Cabello. ${ }^{60}$

Si tomamos en cuenta, como señala Eduardo Báez, que durante el primer año las obras consistieron en demoler el edificio anterior, abrir los cimientos para el nuevo y terraplenar el terreno, ${ }^{61}$ realmente parecería que la participación de Diego de los Santos fue de poca monta. Sin embargo, es casi seguro que la elaboración del proyecto para la nueva fábrica se debió a ambos maestros, por más que correspondiera culminarla solo a Feliciano Cabello. ${ }^{62}$

La última noticia de este maestro es el avalúo que hiciera de una celda en el convento de San Lorenzo, el 6 de septiembre de 1712, escasos dos meses antes de su muerte. ${ }^{63}$

No quisiera terminar esta revisión sin antes llamar la atención sobre unos puntos, acaso intrascendentes, pero que futuras investigaciones se encargarán de manejar en su justa medida. Uno es el relativo a la relación que al parecer existió entre los dos arquitectos y la orden de Nuestra Señora de la Merced. Recuérdese que las ceremonias de bautizo de dos hijas del primero fueron presididas por miembros destacados de dicha

${ }^{57}$ Ibidem, p 96

${ }^{58}$ Eduardo Báez Macías, op. cit., pp. 32-33

${ }^{59}$ Seguramente se trata de nuestro arquitecto, por más que Báez apunta que quedaron dirigiendo la obra Feliciano Cabello y "Antonio" de los Santos; ibidem, p. 33.

${ }^{60}$ Ibidem, p. 34

${ }^{61}$ Ibidem, p. 33.

${ }^{62}$ Martha Fernández, op cit., pp 198 y 277

${ }^{63} \mathrm{Ibidem}$, pp. 198 y 277. 
comunidad, y que tiempo después aparece el hijo acordando con otros maestros la ejecución de algunas obras menores en ese convento. Por ahora no se puede avanzar más, pero si la relación realmente existió, convendría tener presentes a ambos arquitectos a la hora de intentar buscar a quienes intervinieron en la construcción o en algún tipo de reparo del convento o la iglesia de La Merced.

Por otro lado, en un mundo tan pequeño y cerrado como seguramente era el de la Nueva España en esa época, conformado por una comunidad en la que prácticamente todos se conocían, no extraña encontrar al célebre Pedro de Arrieta como testigo en el tercero de los matrimonios de Diego de los Santos, hijo, ni a Felipe de Roa aceptar llevar a la pila bautismal a una hija suya, pero es preciso caer en la cuenta de que tales hechos bien pudieran encerrar un significado de más alcance-verbigracia desde una mera amistad hasta una relación "maestro-alumno"- que el que pudiéramos pensar si sólo apreciáramos la simple relación entre conciudadanos que practicaran un mismo oficio.

Tras estas noticias, creo que la personalidad e importancia de los dos arquitectos que llevaron el nombre de Diego de los Santos y Ávila se nos entrega más coherente y definida. 